\title{
ANÁLISIS DE LAS PROPIEDADES PSICOMÉTRICAS DE LA DIABETES EMPOWERMENT SCALE (DES-LF-S) EN ESPAÑOL
}

\section{ANALYSIS OF THE PSYCHOMETRIC PROPERTIES OF THE SPANISH VERSION OF THE DIABETES EMPOWERMENT SCALE (DES-LF-S)}

\section{TÍTULO CORTO: ANÁLISIS DE LAS PROPIEDADES PSICOMÉTRICAS DE LA DIABETES}

\author{
Patricia Balcázar-Nava ${ }^{1}$, Gloria Margarita Gurrola-Peña ${ }^{2}$, Alejandra Moysén-Chimal ${ }^{3}$, \\ Saúl Urcid-Velarde ${ }^{4}$, Julieta C. Garay-López ${ }^{5}$, Miriam Carolina Velasco-Fuentes ${ }^{6}$, \\ Martha Cecilia Villaveces-López ${ }^{7}$, Elizabeth Estrada-Laredo ${ }^{8}$
}

Recibido en septiembre 01 de 2015

Aceptado en marzo 18 de 2016

\section{RESUMEN}

El propósito de esta investigación fue analizar las propiedades psicométricas de la Escala de Autoeficacia para la Diabetes, Versión Larga en español (DES-LF-S), con 528 adultos con diabetes. El análisis factorial confirmatorio revela que el modelo que mejor se ajusta a los datos en la muestra tiene una estructura de 3 factores y replica la estructura de la escala en su versión original. Los reactivos que componen la escala explicaron el $63.79 \%$ de la varianza. La escala demostró niveles satisfactorios de consistencia interna, obteniéndose un alfa de Cronbach de .96; adicionalmente se obtuvieron los percentiles para la interpretación de resultados individuales. Los hallazgos apoyan el uso de la DES-LF-S para evaluar la autoeficacia relacionada con la diabetes en adultos con la enfermedad.

Palabras clave: Autoeficacia; psicometría; adulto; diabetes mellitus

\section{ABSTRACT}

The purpose of this research was to analyze the psychometric properties of Anderson, Funnell, Fitzgerald y Marrero's Diabetes Empowerment Scale, Long Form-Spanish (DES-LF-S), in 528 diabetic adults. The confirmatory factor analysis stated that the model that best adjust to the data has a tridimensional structure and replicates the structure of the scale in its original version. Items of the scale explained $63.79 \%$ of variance. The scale showed satisfactory

1. Dra. Inv. Psicológica, investigadora, Universidad Autónoma del Estado de México. Correo: pbalcazarnava@hotmail.com

2. Dra. Inv. Psicológica, investigadora, Universidad Autónoma del Estado de México, México. Correo: mgurrolaunid@hotmail.com

3. Dra. Inv. Psicológica, investigadora, Universidad Autónoma del Estado de México, México. Correo: amoysen@hotmail.com

4. Mtro. Psic. Salud, docente, Universidad Autónoma del Estado de México, México. Correo: salulurcid@yahoo.com.mx

5. Dra. Inv. Psicológica, investigadora, Universidad Autónoma del Estado de México, México. Correo: julieta_garay@yahoo.com

6. Lic. Psic. y Lic. Nut., Mtra. en Psic, Universidad Autónoma del Estado de México, México. Correo: mirisvf@hotmail.com

7. Dra. Psic. Lab; investigadora, Universidad Autónoma del Estado de México, México. Correo: mcvillavecesl@uaemex.mx

8. Mtra. Educ., investigadora, Universidad Autónoma del Estado de México, México. Correo: eestradal@uaemex.mx 
levels of internal consistency, obtaining a Cronbach's Alpha of .96; additionally percentiles for the interpretation of individual results were obtained. These findings support the use of DES-LF-S to evaluate self-efficacy related to diabetes in adults.

Keywords: Self-efficacy; psychometry; adult; diabetes mellitus

\section{INTRODUCCIÓN}

$\mathrm{E}_{\mathrm{B}}^{1}$ constructo de autoeficacia fue introducido por Bandura, es un concepto útil en la investigación que permite entender, por ejemplo, el éxito en áreas como desempeño escolar o laboral, disminución de conductas indeseables o cuidado de la salud y adherencia al tratamiento.

Las creencias que las personas tienen acerca de la eficacia para controlar los sucesos en su vida son cruciales para determinar de qué forma hacen frente a problemas y situaciones que en diferentes contextos se le presentan.

Bandura ${ }^{1}$ indica que tanto motivación como conducta están reguladas por el pensamiento e incluyen tres tipos de expectativas: 1) Expectativas de Situación;2) expectativas de Resultado y 3) expectativas de Autoeficacia o Autoeficacia Percibida, que es la que en este trabajo es un concepto central y que se define por Bandura como la creencia que la persona tiene de poseer capacidades para desempeñar acciones necesarias para poder obtener resultados deseados ${ }^{2}$.

El concepto de autoeficacia destaca que la confianza de la persona para desempeñarse en un área determinada; la autoeficacia resulta ser un predictor del éxito, más que las habilidades reales en sí. Esto es, las creencias en sí mismo, ayudan a la persona a tomar decisiones sobre qué hacer con conocimientos y habilidades que poseen ${ }^{1}$.

Las creencias en relación con la eficacia de uno mismo, se desarrollan de 4 fuentes posibles ${ }^{1}$ :

1. Experiencias de dominio: Una experiencia de éxito genera creencias sobre la eficacia personal, mientras que experiencias de no éxito contribuyen a generar una sensación de fracaso, que debilitan estas creencias. Una vez que la persona está convencida de que puede tener éxito, persevera ante la adversidad y se recupera de los problemas.

2. Experiencias vicarias: Se refieren a la observación de personas que se denominan "modelos sociales", quienes alcanzan el éxito tras realizar esfuerzos de forma perseverante. Al observar a estos modelos, la persona genera la expectativa de que perseverar es una estrategia eficaz y de igual forma, al ver a modelos sociales que fracasan, el observador tendrá una predicción de ineficacia.

3. Persuasión social: Se favorece el éxito para realizar algo al decirle a la persona que cuenta con habilidades y herramientas que son necesarias para la realización exitosa de determinadas actividades; la persona fortalece sus creencias de eficacia, se aumenta la probabilidad de que se sienta más segura de sí y se incrementa su movilidad.

4. Estados psicológicos y emocionales: Los estados positivos ayudan a reducir el estrés, favorecen el estado físico y evitan o disminuyen los estados emocionales negativos; la explicación a lo anterior es que la persona en un estado de estrés y de tensión, interpreta una ejecución pobre como una señal de vulnerabilidad.

De acuerdo con su teoría de origen, la autoeficacia es un predictor importante en la forma como la gente siente, piensa y actúa, por lo que un sentido bajo de autoeficacia se asocia con depresión, ansiedad o desamparo; un sentido alto puede hacer que la persona tenga cierto grado de optimismo en los cursos de acción que elige para lograr sus metas y le vuelve autoestimulante ${ }^{3}$.

La autoeficacia ha sido relacionada con diferentes variables, por ejemplo positivamente al bienestar vinculado al comportamiento saludable ${ }^{4}$, negativamente con la depresión ${ }^{5}$; es factor mediador en el éxito de los tratamientos para reducir y mantener la pérdida de peso 0 predictor del abandono en tratamiento de ingesta excesiva $^{6}$; correlaciona con apoyo social y calidad de 
vida en adolescentes con enfermedades crónicas ${ }^{7}$ y con el empoderamiento en las mujeres en etapa de climaterio, para que ejerzan un cuidado efectivo de su salud en esta etapa ${ }^{8}$.

La autoeficacia permite explicar el manejo de enfermedades crónicas como la hipertensión, problemas renales que requieren de hemodiálisis, tratamientos para el VIH-SIDA, variedades de cáncer y diabetes entre otras, al facilitar la adherencia al tratamiento ${ }^{3,9,14}$.

La literatura indica que una enfermedad crónica es una condición de salud de duración extendida en el tiempo mayor a 6 meses y en muchos casos de por vida. En el caso de la Diabetes Mellitus (DM), se trata de un grupo heterogéneo de trastornos metabólicos caracterizados por concentraciones elevadas de glucosa en sangre ${ }^{7}$, que requiere de un manejo integral para su control: es una enfermedad crónica en incremento, en gran parte por el factor genético y también por los altos índices de obesidad y sedentarismo, aspectos del estilo de vida que están asociados con su aparición, su agravamiento y su tratamiento ${ }^{15}$.

La Diabetes Mellitus (DM) se clasifica de acuerdo a su etiología y edad de inicio en tipo uno y tipo dos, en la DM1 existe destrucción de células beta del páncreas, generando deficiencia absoluta de insulina, por lo que suele ser de comienzo abrupto con signos y síntomas de insulinopenia, y es más frecuente en personas menores de 30 años, mientras que en la DM2 existe resistencia a la insulina, hay concomitantemente una deficiencia en su producción, ya sea de forma relativa o absoluta y la diabetes se diagnostica generalmente después de los 30 años de edad ${ }^{16}$. Además de la diabetes tipo 1 y tipo 2, existen algunos otros como la diabetes gestacional y las de tipo Mody, aunque la más frecuente es la DM2.

La DM2 afecta al 9.2\% (6.4 millones de personas) de la población mexicana entre los 20 y los 69 años de edad; es el segundo lugar en defunciones por año según la Encuesta Nacional de Salud y Nutrición (ENSANUT) ${ }^{17}$ y la tasa de mortalidad va en ascenso $0^{16}$ con más de 60 mil muertes anuales según el Sistema Nacional de Información en Salud (SINAIS) y una incidencia mayor a 400,000 casos en el mismo período ${ }^{17}$, por lo que la intervención multidisciplinaria en el caso de esta enfermedad es necesaria y urgente.

Para retrasar o evitar las complicaciones propias de la DM2 (lesiones microvasculares que provocan pérdida visual, neuropatía periférica y central, dificultad en cicatrización, problemas renales y amputaciones), se requiere un plan de tratamiento basado en aspectos que incluyen el farmacológico (medicamentos orales o insulina), alimentación equilibrada en cantidad y calidad, actividad física programada, automonitoreo de glucosa y cuidado general de órganos y sistemas bajo supervisión médica especializada.

Para el tratamiento de la DM2 es necesario un equipo interdisciplinario que pueda ayudarle al paciente al cumplimiento de las metas en su tratamiento, entre ellas, conocimientos para el manejo de la enfermedad, cambio en las creencias sobre salud y enfermedad, dominio de habilidades diversas para hacer frente a la DM2 y un alto grado de motivación para el logro del tratamiento, por lo que la autoeficacia permite explicar el logro de la adherencia al tratamiento y es un buen indicador del éxito o fracaso.

En el caso de la salud, la autoeficacia es una variable que ayuda a predecir su cuidado y las decisiones sobre las cuales se fundamentan los estilos de vida. Desde el enfoque de Bandura ${ }^{8}$, la autoeficacia es crucial para entender cómo una persona puede empoderarse y tomar así decisiones informadas acerca de sí y le convierte en un agente activo en el cuidado de su salud.

Anderson y su equipo de trabajo desarrollaron la Diabetes Empowerment Scale (DES). La versión original en inglés de la DES-LF ${ }^{9}$ se ha traducido y/o adaptado en diferentes idiomas $\left(\right.$ chino $^{18}$, sueco ${ }^{19}$, versión corta de la DES en inglés ${ }^{20}$ ), presentando valores alfa de Cronbach entre .86 y .96, que demuestran sus propiedades.

Aun cuando existía una versión en español, se hizo necesaria su adaptación al contexto local, pues originalmente había sido traducida y probada en grupos con idioma español, en población hispana que vive en Estados Unidos y dadas las condiciones geográficas y culturales, el lenguaje podría tener variaciones en el contexto de México.

La idea de retomar la DES es que en primer lugar, se están haciendo esfuerzos por atender a la DM2 de forma integral, lo que incluye una serie de programas basados en la educación diabetológica, mismos que buscan el empoderamiento del paciente. Para poder identificar la efectividad de los programas se evalúa la autoeficacia en diferentes momentos, por ejemplo al inicio y al final de las intervenciones; en segundo lugar, se buscó literatura 
sobre estas escalas y se encontró que la DES tiene un buen comportamiento psicométrico en otros idiomas y al revisar lo existente en México, los resultados de la búsqueda indicaron la existencia de una escala ${ }^{21}$ para pacientes con DM2, sólo que evalúa la eficacia centrada en aspectos específicos del tratamiento (plan alimenticio, actividad física y toma adecuada de medicamentos) y no contempla una idea general de las creencias de autoeficacia, por lo que se decidió trabajar en la adaptación de la DES-LF-S en muestras mexicanas.

Debido a la relevancia de la autoeficacia en los procesos crónicos y a la gran prevalencia del padecimiento en México, es necesario contar con instrumentos psicométricamente adecuados y adaptados a la población que posibiliten la investigación y el manejo terapéutico integral de los pacientes.

El objetivo de esta investigación fue analizar las propiedades psicométricas de la DES-LF-S en una muestra de pacientes con DM2 de la ciudad de Toluca, Estado de México. Los objetivos específicos fueron obtener su validez de constructo, su consistencia interna y los baremos para su interpretación.

\section{MATERIALES Y MÉTODOS}

\section{Tipo de estudio y participantes}

Es un estudio de tipo Instrumental, que se utiliza cuando se diseñan o se adaptan pruebas y aparatos ${ }^{22}$. Para este estudio, la obtención del número de participantes se realizó atendiendo a consideraciones ${ }^{23}$, de que el mínimo necesario para proporcionar suficiente estabilidad al análisis es de 200 personas; también se tomó en cuenta lo indicado por Carretero-Dios y Pérez ${ }^{24}$, quienes proponen que en los análisis de tipo factorial confirmatorio, deberá considerarse una muestra entre 5 y 10 individuos por cada ítem. Los criterios de inclusión de los participantes fueron: personas adultas, con diagnóstico de DM2, mínimo un año de haber sido diagnosticado con la enfermedad, que superan leer y escribir, que en el momento de la aplicación no tuvieran complicaciones agudas derivadas de la DM2 y que aceptaran participar de forma voluntaria.

\section{Instrumento}

La DES-LF-S ${ }^{9}$ integra 28 reactivos, en escala tipo Likert de 5 gradaciones (" 5 , Totalmente de acuerdo" hasta " 1 , Totalmente en desacuerdo"). Es una medida elaborada en el Michigan Diabetes Research and Training Center, para evaluar autoeficacia relacionada con el cuidado de DM2 en contextos de tipo educacional y de intervención psicosocial; desarrollada con la premisa de que el paciente es responsable en gran medida, de su cuidado y que aquéllos más autoeficaces toman decisiones que afectan y que son afectadas por aspectos psicosociales de vivir con una enfermedad crónica. Ante la falta de instrumentos que midieran autoeficacia, los autores desarrollaron la DES, que originalmente contenía 37 reactivos agrupados en 8 subescalas; tras numerosos análisis quedaron 28 ítems que explicaban el $55 \%$ de la varianza total, alfa de .96 y con 3 subescalas: 1) Manejando los aspectos psicosociales de la diabetes (9 reactivos, alfa $=.93$ ); 2) Evaluando la insatisfacción y la disposición al cambio ( 9 reactivos, alfa $=.81)$ y 3 ) Fijando y logrando las metas en diabetes (10 reactivos, alfa $=.91$ ). Tiene formato autoadministrable, a personas mínimo con educación primaria; la puntuación total se obtiene promediando los puntajes de los 28 ítems; puntajes altos indican mayores niveles de autoeficacia.

\section{Procedimiento}

Se aplicó la DES-LF-S a los participantes explicándoles el objetivo de la investigación y solicitándoles autorización para aplicar la escala. A cada participante se le explicó que su colaboración era voluntaria, anónima y que no existían respuestas buenas o malas, la prueba se aplicó de manera individual, mientras un profesional de la psicología estuvo presente durante su resolución, por si existía alguna duda y luego fue devuelta a los investigadores para su análisis.

\section{Análisis de los Datos}

Con el fin de determinar la validez de constructo y la confiabilidad de la escala al utilizarla en el contexto local, se realizó un análisis factorial; el análisis de la consistencia interna se realizó a través de la obtención del alfa de Cronbach, además de la transformación de puntajes totales de la DES-LF-S a percentiles. Se empleó el software estadístico para las Ciencias Sociales SPSS, versión 21 con licencia de utilización para la Universidad Autónoma del Estado de México (UAEMex).

\section{Declaración de aspectos éticos}

Los participantes fueron informados del objetivo de la investigación, aceptaron participar voluntariamente, para lo que firmaron una carta de consentimiento informado por duplicado, una que les fue devuelta a ellos y otra que el equipo de investigación retuvo, que iba acorde con los principios y normas éticas de la Declaración de Helsinki. 


\section{RESULTADOS}

\section{Análisis Factorial Exploratorio y Confirmatorio}

Se aplicó la escala a una muestra intencional de 528 personas con DM2; de ellos, 181 eran hombres y 347 mujeres, con edades entre 35 y 70 años; el intervalo con DM2 fue entre uno y más de 10; 437 participantes manifestaron tener plan de tratamiento para su DM2 y el resto, no.

En primer término, se evaluó si las matrices de correlaciones eran adecuadas, por lo que se utilizó el índice de Kaiser-Meyer-Olkin $(\mathrm{KMO}=.954)$ y la prueba de Bartlett $\left(X^{2}=3767.756, p<.00\right)$. Estas medidas dieron cuenta de una buena adecuación muestral, además de lo pertinente del análisis factorial y que no existía esfericidad en los datos.

Los 28 ítems de la DES-LF-S se sometieron a un análisis factorial exploratorio, mediante el método de componentes principales con rotación varimax. Para poder determinar los factores que de manera inicial se tomarían, se utilizó el criterio de extracción basado en el gráfico de sedimentación de Cattell ${ }^{6}$, que sugiere la presencia de 3 factores que cubren el criterio de valores Eigen iguales o superiores a uno (Figura 1).

Posteriormente, se realizó otro análisis de tipo confirmatorio, que se forzó a tres factores atendiendo al número de factores de la escala en su versión original. Algunos de los criterios para poder seleccionar e interpretar los factores que integrarían la prueba entre otros son: valores Eigen iguales o mayores a uno y al menos tres reactivos en cada factor. Adicionamente, para decidir si un reactivo pertenece a un factor, se consideró como criterio que su carga factorial fuese de .40 o más, que ésta tuviese un desarrollo positivo y si un reactivo cargaba en dos o más factores, se asignaría a aquél con peso factorial mayor.

Se analizaron los pesos factoriales de los reactivos en cada uno de los tres factores. Tal y como puede apreciarse en la Tabla 1, cuatro de ellos (los reactivos 5, 15, 16 y 26), tenían pesos compartidos, considerando un peso compartido, cuando la diferencia entre las dos cargas es menor a 10.

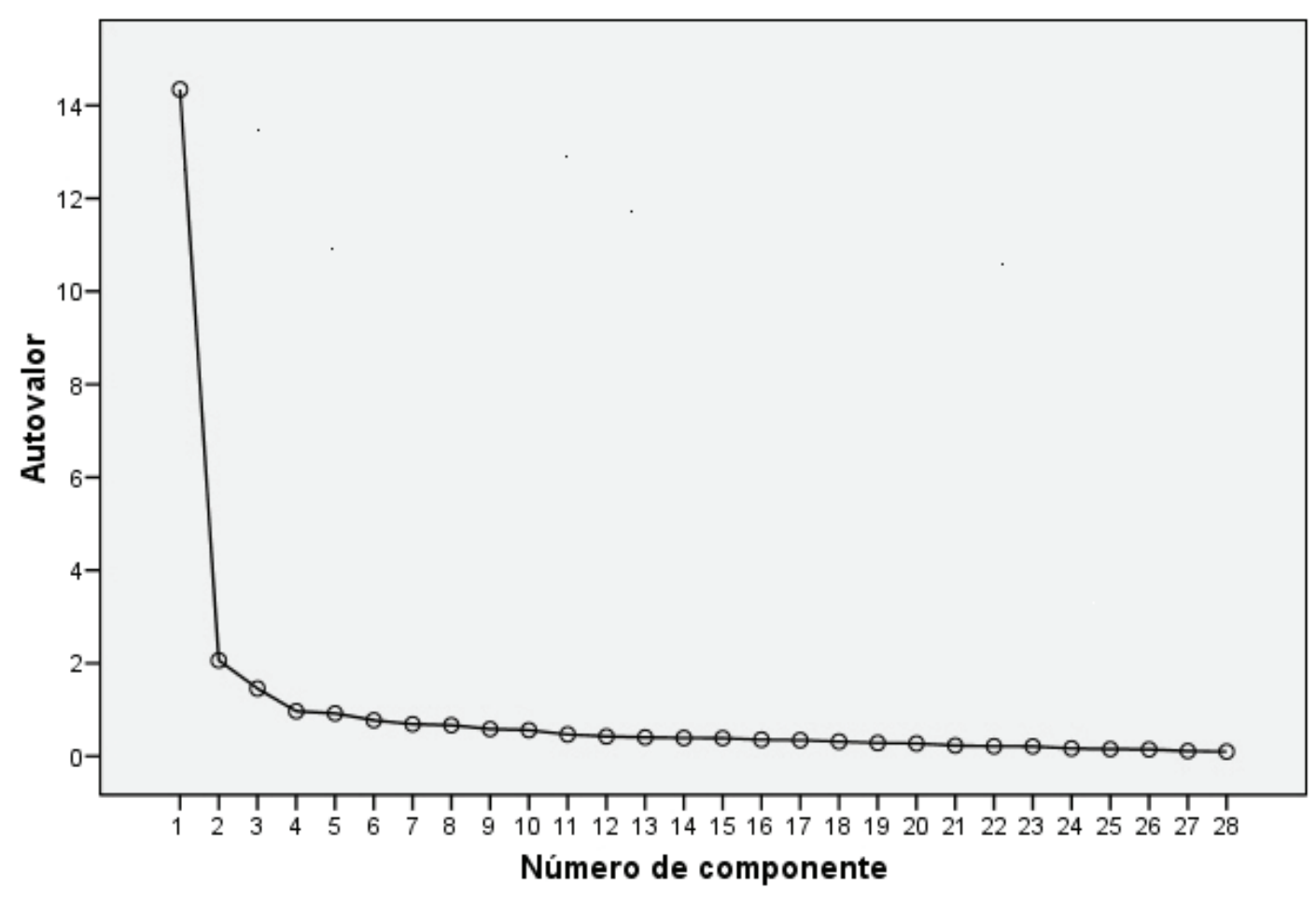

Figura1. Sedimentación DES-LF-S aplicada a 528 participantes con DM2.2015 
ANÁLISIS DE LAS PROPIEDADES PSICOMÉTRICAS DE LA DIABETES EMPOWERMENT SCALE (DES-LF-S) EN ESPAÑOL

Tabla 1. Resumen de reactivos y cargas factoriales para la solución ortogonal de la DES-LF-S aplicada a 528 participantes con DM2-2015

\begin{tabular}{|c|c|c|c|c|}
\hline \multicolumn{5}{|c|}{ Carga factorial } \\
\hline Reactivo & Factor 1 & Factor 2 & Factor 3 & Comunalidad (h2) \\
\hline 22 & .82 & & & .75 \\
\hline 25 & .78 & & & .77 \\
\hline 21 & .75 & & & .64 \\
\hline 24 & .72 & .42 & & .72 \\
\hline 23 & .69 & & & .63 \\
\hline 10 & .67 & .49 & & .69 \\
\hline 9 & .66 & .49 & & .68 \\
\hline 27 & .63 & & & .63 \\
\hline 16 & .60 & .57 & & .73 \\
\hline 15 & .60 & .56 & & .71 \\
\hline 28 & .59 & & .41 & .57 \\
\hline 26 & .58 & & .47 & .58 \\
\hline 4 & & .71 & & .55 \\
\hline 13 & .43 & .66 & & .69 \\
\hline 1 & & .66 & & .60 \\
\hline 8 & .42 & .66 & & .65 \\
\hline 11 & & .66 & & .52 \\
\hline 14 & .45 & .65 & & .70 \\
\hline 2 & & .64 & & .48 \\
\hline 12 & .43 & .62 & & .63 \\
\hline 7 & .49 & .62 & & .63 \\
\hline 3 & & .60 & & .52 \\
\hline 6 & .56 & .58 & & .65 \\
\hline 5 & .49 & .57 & & .57 \\
\hline 18 & & & .83 & .77 \\
\hline 19 & & & .78 & .65 \\
\hline 20 & & & .77 & .67 \\
\hline 17 & & & .61 & .47 \\
\hline
\end{tabular}

Nota: Las cifras en negritas indican cargas factoriales más altas.

Como se aprecia en la Tabla 1, el rango de los pesos factoriales de los reactivos estuvo entre .57 y .83 ; el primer factor explica el $51.23 \%$ de la varianza total; 11 reactivos conforman este factor, con pesos factoriales entre $.58 \mathrm{y}$ .82 , cuya temática se relaciona con el Manejo de Aspectos
Psicosociales de la Diabetes. El segundo factor explica un $7.36 \%$ de la varianza, con 12 reactivos con pesos factoriales entre .57 y .71, referidos a Metas y Barreras en el Cuidado de la Diabetes. El tercer factor, explica un $5.20 \%$ de la varianza y se compone de cuatro reactivos 
con pesos factoriales entre .61 y .83 y que exploran la Identificación y Manejo de Estrés. En su conjunto, los 28 reactivos de la DES-LF-S, explican el $63.8 \%$ de la varianza total.

\section{Fiabilidad}

Se calculó el índice de consistencia interna (coeficiente alfa de $C$ ronbach $=\alpha$ ) para cada factor y para la escala total. Los valores alpha de los factores son: Factor 1 $\alpha=.95$, Factor $2 \alpha=.94$ y Factor $3 \alpha=.83$. Para la escala total el valor fue $\alpha=.96$; con base en los hallazgos, se observan niveles de fiabilidad satisfactorios en los reactivos que integran a cada uno de los tres factores y a la escala en general. Adicionalmente, se obtuvieron los valores para poder interpretar los puntajes de la escala al trabajarse en investigación, mediante percentiles (Tabla 2).

Tabla 2. Normas de la DES-LF-S para muestras mexicanas de personas con DM2

\begin{tabular}{|c|c|c|}
\hline Percentiles & $\begin{array}{c}\text { Puntaje total de } \\
\text { la DES-LF-S }\end{array}$ & Interpretación \\
\hline $1-20$ & $28-50$ & Bajo \\
\hline $21-40$ & $51-57$ & Por debajo del promedio \\
\hline $41-60$ & $58-61$ & Promedio \\
\hline $61-80$ & $62-68$ & Por arriba del promedio \\
\hline $81-100$ & $69-140$ & Alto \\
\hline
\end{tabular}

Fuente: Elaboración propia con los datos del estudio.

\section{DISCUSIÓN}

La DES, en su versión original, ha demostrado ser una medida válida y confiable para evaluar autoeficacia en una variedad de contextos educacionales y de intervención psicosocial relacionados con la DM2 y de la que se editaron una forma larga y otra corta, en idiomas diferentes incluyendo español, por lo que se decidió utilizarla en virtud del peso que el constructo tiene en la atención de la $\mathrm{DM}^{9,11}$, que permite explicar los cambios efectuados y el éxito en su tratamiento.

La revisión de la literatura sobre la DES-LF-S en diversas bases científicas de datos, permitió concluir que no había datos de su utilización en muestras mexicanas con base en el modelo de autoeficacia, propuesto teóricamente por Bandura y que este constructo tiene una especificidad dependiendo del contexto en el cual se desee evaluar la autoeficacia, lo cual justifica su adaptación en poblaciones atendiendo a contextos específicos.

En su versión original ${ }^{9}$, la DES es una medida que integra tres áreas para evaluar la autoeficacia relacionada con el manejo de la Diabetes Mellitus 2. Resulta de importancia indicar que el uso de este tipo de medidas es crucial cuando se desea llevar a cabo alguna experiencia relacionada con el tratamiento de la DM2 y contar con una medida que esté adaptada a muestras locales es un paso previo a estos programas de intervención. Los resultados de la adaptación de la DES-LF-S en un primer análisis de tipo exploratorio, tras verificar la gráfica de sedimentación, indicaron que la Escala presentaba una estructura que podía someterse a un análisis forzado de tres factores, acorde con el modelo original de la DES y de la propuesta teórica del constructo de autoeficacia.

Los resultados de los alfas de Cronbach son similares a los obtenidos por los creadores de la DES-LF-S (.96) y en su adaptación a otros idiomas (entre .89 y .96) ${ }^{9}$, que revelan una alta consistencia interna. Lo anterior indica que la DES-LF-S tiene un alto grado de estabilidad, precisión o consistencia como instrumento de medición de la autoeficacia relacionada con la diabetes; este alto valor del coeficiente indica que en su conjunto los 28 reactivos miden el constructo estudiado con una covariación fuerte entre $s^{\prime 2}$.

Al contrastar el modelo propuesto por los creadores de la DES-LF-S, se observa que el análisis factorial en esta investigación agrupa los 28 reactivos originales en su totalidad. Resaltan las cualidades de la escala con valores Eigen superiores a uno y que en su conjunto explican un alto porcentaje de varianza total, dato que indica la 
cantidad de varianza de una variable o constructo que queda explicada por los reactivos de un instrumento, demostrando así una adecuada explicación del constructo de referencia y que se corrobora también con los datos psicométricos de la DES en su versión corta (DES-SF) ${ }^{20}$. En este caso, puede argumentarse lo que indica la revisión ${ }^{24}$, de que los reactivos de la prueba son indicadores observables de un constructo que subyace una teoría y que su comprobación empírica mediante procedimientos estadísticos permite indicar si son adecuados, por lo que se concluye que los 28 reactivos son coherentes con el modelo teórico sobre la autoeficacia relacionada con la diabetes.

El análisis de lo que en su conjunto agrupan los reactivos de las tres sub-escalas permitió asignarles nombre, observándose la consistencia de los reactivos para evaluar cada una de las partes integrantes de la autoeficacia en el cuidado de la diabetes, mismos que se nombraron acorde a lo que contenían en su conjunto.

En lo que respecta a los indicadores que componen el constructo de autoeficacia, Anderson et $\mathrm{al}^{9}$, sugerían cuatro pasos que integran el modelo de cambio conductual: 1) identificación de áreas problemáticas por el paciente; 2) exploración de emociones asociadas con esos problemas; 3) desarrollo de un conjunto de metas y estrategias para vencer las barreras y lograr las metas y 4) hacer que exista un plan para cambiar conductas a través de la motivación. Con base en ese modelo, se construyó la DES, que tras arreglos y numerosas aplicaciones y experiencias prácticas de manejo en pacientes con diabetes, se concluye que tanto el manejo de estrés como el soporte psicosocial que la persona puede obtener de quienes le rodean, podrían integrarse en una sub-escala que derivaría en barreras y/o facilitadores del cambio conductual, así como en una adaptación psicosocial a la diabetes, por lo que la DES en su versión inicial se integró en 3 sub-escalas y en este estudio, tras analizar la versión en español, reproduce una estructura similar.

Por otra parte, con el uso de las escalas interesa estudiar el grado en que las puntuaciones de un cuestionario o escala sirven para clasificar y comparar esas puntuaciones que obtienen diferentes sujetos dentro de ciertos grupos de referencia ${ }^{24}$. Conforme a la literatura consultada, una de las formas en las cuales se pueden obtener baremos de las pruebas es través del uso de centiles, que representan una medida de posición en una distribución de frecuencias ${ }^{24}$.

Los centiles o percentiles permiten indicar en un valor del 0 al 100 la puntuación obtenida por una persona con respecto a un grupo normativo, por lo que además de comprobar las propiedades psicométricas de la DES-LF-S, se obtuvieron los percentiles con la finalidad de facilitar la interpretación de puntuaciones individuales en el grupo de referencia.

\section{CONCLUSIÓN}

La primera conclusión apunta a que la DES-LF-S agrupa componentes relacionados con el manejo de la diabetes, ajustándose al modelo teórico de base sobre autoeficacia. La segunda conclusión es la que hace referencia a que la DES-LF-S cumple los criterios psicométricos que la hacen adecuada para su utilización en muestras de mexicanos del centro del país, demostrando su fuerte consistencia interna, al poseer validez y confiabilidad altas para ser utilizada con toda confianza en la investigación.

Finalmente, se concluye que la DES-LF-S puede ayudar en la identificación y clasificación de personas con diabetes mellitus en relación con la autoeficacia que perciben en el cuidado de su enfermedad y podría utilizarse en diferentes momentos con el paciente, por ejemplo antes y después de una intervención para el logro de la adherencia al tratamiento, para ver las fluctuaciones de la autoeficacia en diferentes momentos.

\section{DECLARACIÓN SOBRE CONFLICTOS DE INTERÉS}

Los autores manifiestan que este producto final de investigación no fue financiado o apoyado económicamente y que no existen conflictos de intereses o valores.

\section{REFERENCIAS BIBLIOGRÁFICAS}

1. Bandura A. Self-efficacy: the exercise of control. $1^{\text {st }}$ Edition. New York: Freeman Editorial; 1997.

2. Bandura A. Self-efficacy mechanism in human agency. $1^{\text {st }}$ Edition. New York: Cambridge University Press; 1995.

3. Olivari-Medina C, Urra-Medina E. Autoeficacia y conductas de salud. Ciencia y enfermería [revista en la Internet]. 2007 [citado 2015 marzo 15]; 13(1): 9-15. Disponible en: http://www.scielo.cl/scielo.php? script $=$ sci_ arttext\&pid $=$ S0717-95532007000100002\&lng $=$ es

4. Reyes-Jarquín K, Hernández-Pozo MDR. Análisis crítico de los estudios que explican la autoeficacia y bienestar vinculados al comportamiento saludable. Journal of Behavior, Health \& Social Issues [revista en la Internet]. 2011 [citado 2015 enero 19]; 3(2): 5-24. Disponible en: http://www.redalyc.org/articulo.oa?id $=282221802002$ 
5. Díaz-Santos M, Cumba-Avilés E, Bernal G, RiveraMedina C. Desarrollo y propiedades psicométricas de la Escala de Autoeficacia para la Depresión en Adolescentes (EADA). Interamerican Journal of Psychology [revista en la Internet]. 2008 [citado 2015 enero 19]; 42(2): 218227. Disponible en: http://www.redalyc.org/articulo. oa?id $=28442204$

6. Román Y, Díaz B, Cárdenas M, Lugli Z. Construcción y validación del Inventario Autoeficacia Percibida para el Control de Peso. Clínica y Salud [revista en la Internet]. 2007 [citado 2015 enero 30]; 18(1): 45-56. Disponible en: http://www.copmadrid.org/webcopm/resource. do? recurso $=4000 /$ \&numero $=2007181$

7. Avendaño Monje MJ, Barra Almagiá E. Autoeficacia, apoyo social y calidad de vida en adolescentes con enfermedades crónicas. Terapia Psicológica [revista en la Internet]. 2008 [citado 2015 marzo 22]; 26(2): 165172. Disponible en: http://www.redalyc.org/articulo. oa? id $=78511540002$

8. Doubova SV, Espinosa-Alarcón P, Infante C, AguirreHernández R, Rodríguez-Aguilar L, Olivares-Santos R, et al. Adaptación y validación de escalas de autoeficacia y empoderamiento dirigidas a mujeres mexicanas en etapa de climaterio. Salud pública de México [revista en la Internet]. 2013 [citado 2015 febrero 02]; 55(3): 257266. Disponible en: http://www.redalyc.org/articulo. oa? id $=10628332003$

9. Anderson RM, Funnell MM, Fitzgerald JT, Marrero DG. The Diabetes Empowerment Scale: A measure of psychosocial self-efficacy. Diabetes Care [revista en la Internet]. 2000 [citado 2011 noviembre 19]; 23(6): 739743. Disponible en: http://care.diabetesjournals.org/ content/23/6/739.long

10. Ortiz M, Ortiz E. Adherencia al tratamiento en adolescentes diabéticos tipo 1 chilenos: Una aproximación psicológica. Revista médica de Chile [revista en la Internet]. 2005 [citado 2011 diciembre 16]; 133(3): 307-313. Disponible en: http://dx.doi. org/10.4067/S0034-98872005000300006

11. Pérez-Pastén E. Guía para el paciente y el educador en diabetes. Primera edición. México: Editorial Johnson y Johnson; 2006.

12. Oviedo-Gómez MT, Reidl-Martínez LM. Predictores psicológicos individuales de la calidad de vida en diabetes tipo 2. Revista Mexicana de Psicología [revista en la Internet]. 2007 [citado 2015 enero 21]; 24(1): 3142. Disponible en: http://www.redalyc.org/articulo. oa?id $=243020635005$.

13. Álvarez-Mabán E, Barra-Almagia E. Autoeficacia, estrés percibido y adherencia terapéutica en pacientes hemodializados. Ciencia y enfermería [revista en la Internet]. 2010 [citado 2014 diciembre 20]; XVI(3): 63-72. Disponible en: http://www. scielo.cl/scielo.php?script $=$ sci_arttext\&pid $=$ S0717 95532010000300008\&lng = es
14. Pérez-Pedrogo C, Martínez-Taboas A. Factores asociados a la sintomatología depresiva en adultos/as con diabetes y el impacto en su autoeficacia y en el cuidado de la salud. Salud \& Sociedad [revista en la Internet]. 2011 [citado 2015 abril 02]; 2(1): 044-061. Disponible en: http://dialnet.unirioja.es/descarga/articulo/4017543. pdf

15. Instituto Mexicano del Seguro Social. Diagnóstico y tratamiento de la diabetes mellitus tipo 2. México, D.F.: IMSS; 2012.

16. México. Secretaría de Salud. Norma Oficial Mexicana NOM-015-SSA2-2010, Para la prevención, tratamiento y control de la diabetes mellitus [internet]. [Consultado 2016 marzo 12]. Disponible en: http://dof.gob.mx/ nota_detalle.php? codigo $=5168074$ yecha $=23 / 11 / 2010$

17. México. Instituto Nacional de Salud Pública. Encuesta Nacional de Salud y Nutrición [internet]. [Consultado 2016 marzo 12]. Disponible en: http://ensanut.insp.mx/

18. Shiu ATY, Wong RYM, Thompson DR. Developments of a reliable and valid Chinese version of The Diabetes Empowerment Scale. Diabetes Care [revista en la Internet]. 2003 [citado 2012 diciembre 10]; 26(10): 28172821. Disponible en: http://care.diabetesjournals.org/ content/26/10/2817.full

19. Leksell J, Funnell M, Sandberg G, Smide B, Wiklund G, Wikblad K. Psychometric properties of the Swedish Diabetes Empowerment Scale. Diabetes Care [revista en la Internet]. 2007 [citado 2014 diciembre 17]; 21(2): 247-252. Disponible en: http://www.readcube.com/ articles/10.1111\%2Fj.1471-6712.2007.00463.x?r3 referer $=$ wol\&tracking_action $=$ preview_click\&show checkout $=1$ \&purchase_referrer $=$ onlinelibrary . wiley. com\&purchase_site_license = LICENSE_DENIED_NO_ CUSTOMER

20. Anderson RM, Fitzgerald JT, Gruppen LD, Funnell MM, Oh MS. The Diabetes Empowerment Scale-Short Forms (DES-SF). Diabetes Care [revista en la Internet]. 2003 [citado 2012 diciembre 12]; 23(5): 1635-1660. Disponible en: http://care.diabetesjournals.org/ content/26/5/1641.2.long

21. Castillo DA, Guzmán RME, Iglesias S, Reyes I. Validez divergente y convergente del instrumento de autoeficacia al tratamiento en diabetes. Psicología Iberoamericana [revista en la Internet]. 2012 [citado 2013 enero 20]; 20(2): 58-65. Disponible en: http://www.redalyc.org/ articulo.oa? id $=133928816008$

22. Montero I, León OG. Clasificación y descripción de las metodologías de investigación en Psicología. Revista Internacional de Psicología Clínica y de la Salud [revista en la Internet]. 2002 [citado 2015 mayo 22]; 2(3): 503-508. Disponible en: http://www.aepc.es/ijchp/ articulos_pdf/ijchp-53.pdf

23. Nunnally JC. Teoría psicométrica. Segunda edición. México: Editorial Trillas; 1997. 
24. Carretero-Dios H, Pérez C. Normas para el desarrollo y revisión de estudios instrumentales. International Journal of Clinical and Health Psychology [revista en la Internet]. 2007 [citado 2016 marzo 04]; 5(3): 521-551. Disponible en: http://www.aepc.es/ijchp/ NDREI07_es.pdf
25. Abad FJ, Garrido J, Olea J, Ponsoda V. Introducción a la psicometría. Teoría clásica de los tests y teoría de la respuesta al ítem. Primera edición. Madrid, España: Universidad Autónoma de Madrid; 2006.

Para citar este artículo: Balcázar-Nava P, Gurrola-Peña GM, Moysén-Chimal A, Urcid-Velarde S, Garay-López JC, Velasco-Fuentes MC, et al. Análisis de las propiedades psicométricas de la diabetes Empowerment Scale (DES-LF-S) en español. Duazary. 2016 julio; 13 (2): 95 - 104 